\title{
Nudging towards Serendipity: A case with personal digital photos
}

\author{
Tuck Wah Leong \\ Culture Lab, School of Computing Science \\ Newcastle University \\ Newcastle-upon-Tyne NE1 7RU, UK \\ tuck.leong@ncl.ac.uk
}

\author{
Richard Harper \\ Microsoft Research Cambridge \\ $7 \mathrm{JJ}$ Thomson Avenue Cambridge, \\ CB3 OFB. UK \\ r.harper@microsoft.com
}

\author{
Tim Regan \\ Microsoft Research Cambridge \\ 7 JJ Thomson Avenue Cambridge, \\ CB3 OFB. UK \\ timregan@microsoft.com
}

\begin{abstract}
Serendipity is an engaging, deeply personal and even magical experience to some. While serendipity has been noted to arise during people's interactions with digital photos, we have yet to understand how this occurs or how it could be supported during-and-through the use of technology. Inspired by findings about serendipity arising from people's shuffle listening, we designed a digital photo display system to explore how we could support people's encounters with serendipity with digital photos. Through this, we gained a deeper understanding of this technology-mediated serendipity and found ways that can support and even nudge people towards encountering serendipity.
\end{abstract}

Digital photos. Serendipity. Randomness. Defamiliarization. Photo Display System.

\section{INTRODUCTION}

Serendipity is a coined word, created ex nihilo by Horace Walpole in 1754 [Merton \& Barber, 2004]. Walpole fashioned this word to describe accidents or chance encounters that lead to desirable or fortunate discoveries. We see this particularly in the history of science where many accidental (but fortuitous) discoveries have been attributed to serendipity; e.g. Röntgen's discovery of X-rays and Fleming's discovery of penicillin [Roberts, 1989; Austin, 2003]. But while serendipity can afford great insights and discoveries, our encounters with serendipity within the ordinariness of quotidian life can be a "particularly pleasant experience, the more so if it is a significant one" [Merton \& Barber, 2004 , p. 82]. Often, the experience is imbued with magic and wonder, bearing the capacity not only to delight and thrill but also regarded as a product of chance and luck. In fact, serendipitous encounters are among the experiences that people like to remember and report, often to recount their good fortune to others. Thus serendipity is not only a potentially rich experience that can capture people's imagination, it is also personally significant, meaningful and engaging - a human experience highly relevant to Human-Computer Interaction $(\mathrm{HCl})$ in its embrace of the UserExperience (UX) agenda.

Efforts to explore how we can support for the rich and personally meaningful experience of serendipity during-and-through people's use of technology will contribute to a deeper understanding of this phenomenon whilst extending the UX agenda from its current (limited) cast of experiences of fun and enjoyment. For while understanding and supporting design for playfulness, pleasure and enjoyment is certainly a positive step, an assumption that people always want to have fun and be pleased represents a simplistic view of human nature [Petersen et al., 2004]. Furthermore, understanding how to support for this serendipity when designing interactive technologies heeds the call for $\mathrm{HCl}$ researchers and practitioners to look beyond instrumental goals and to consider experiences that are meaningful, and even magical whilst contributing to our sense of self and well being [Harper et al., 2008].

This paper describes our design-led and experience-centered approach to deepen our understanding of this particular technologymediated serendipity. It involved designing a system, deploying it in an empirical study and examining experiential accounts of serendipity during-and-through its use in naturalistic settings. What we designed was a digital Photo Display System (PDS) that allowed people to interact with their personal digital photo collection. This was motivated in part by the fact that serendipity has been reported notably when people interact with their personal digital media collection, such as digital music [Leong, T. et al., 2005] or with digital photos [Shneiderman et al., 2006; Durrant et al., 2009]. Since some studies of such interactions 
have surfaced particular elements that purportedly nudge people towards encountering serendipity, we incorporated these elements into our design. Hence our other aim was to ascertain the veracity of such claims, and in the process, gain further insights into this technology-mediated serendipity.

Next, we will briefly examine relevant and related work before describing the PDS and the rationale behind the design. Following this, we present details of the empirical study whereby the PDS was deployed in three households. Our discussion shows how the various elements embodied by the PDS enriched people's viewing experience and even nudged people towards serendipitous encounters. We close by pointing to potentially fruitful approaches when designing technologies to support people's encounters with serendipity.

\section{RELATED WORK}

Most efforts with regards to digital media and our interactions with it had been either about trying to understand people's practices [Bull, 2006; Czerwinski et al., 2006; Kirk et al., 2006; Shen et al., 2009] or trying to (efficiently and effectively) support people's interactions with digital media. And whilst some have urged the need for systems to support serendipitous discoveries when interacting with digital media [Bentley et al., 2006], we have yet to see efforts that focus explicitly upon serendipity. However, some have offered clues about this technology-mediated serendipity.

Nunes et al's [2009] prototype designed to encourage social and collocated digital photo sharing was found to lead to serendipitous conversation and photos discovery. They reported that the use of personally meaningful material, such as people's physical memorabilia linked to their digital photos supported serendipitous encounters. They also noted the influence of randomness. People reported encountering serendipity during reminiscing that was triggered by randomly selected snippets of their personal content presented by a system [Cosley et al., 2009]. Leong et al's [2005; 2006; 2008a; 2008b] studies of people's shuffle listening experience - a mode of listening whereby listeners abdicate choice to the digital music player to pick tracks randomly revealed a similar but more nuanced picture. They posited that the random presentation of tracks also defamiliarized people's memories and prior associations imbued upon their digital music tracks. Defamiliarization is a process that "makes things unfamiliar, to increase the difficulty and length of their perception" [Shklovsky, 1917]. In fact, Leong et al suggested that the combination of various elements: personally meaningful tracks, the random presentation, the effect of defamiliarization and temporality worked in concert to nudge listeners towards encounters with serendipity.

So, we took Leong et al's [2005; 2006; 2008a; 2008b] findings and used the elements of randomness, defamiliarization and temporality to guide the design of a digital PDS. And by deploying this PDS in homes, we hoped to (i) ascertain the extent that these elements (surfaced through studying shuffle listening) play in influencing people's encounters with serendipity, (ii) establish a deeper understanding of how we could support for serendipitous encounters with digital photos and in turn, (iii) extend our understanding of this technology-mediated serendipity during interactions with digital media.

\section{PDS: PHOTO DISPLAY SYSTEM}

While digital photo displays are available commercially, we designed one that houses a photo presentation application that expresses the particular elements we were interested in. Given that this PDS would 'live' in people's homes, we took great care to ensure that it 'fits' within a domestic setting and how it may be used. Besides the necessary processing power, it had to be small and discrete without overwhelming the domestic space while still providing adequate display visibility. We also designed a novel dice-led device that could be used by people to interact with the PDS. The PDS (fig. 1) consisted of the following:

(i) The photo presentation application running on Windows in a Mac mini computer

(ii) A color monitor (11", 600x800 resolution)

(iii) A dice-led input device composed of a Phidgets RFID 1 tag and reader.

(iv) People's digital photo library housed in the Mac mini

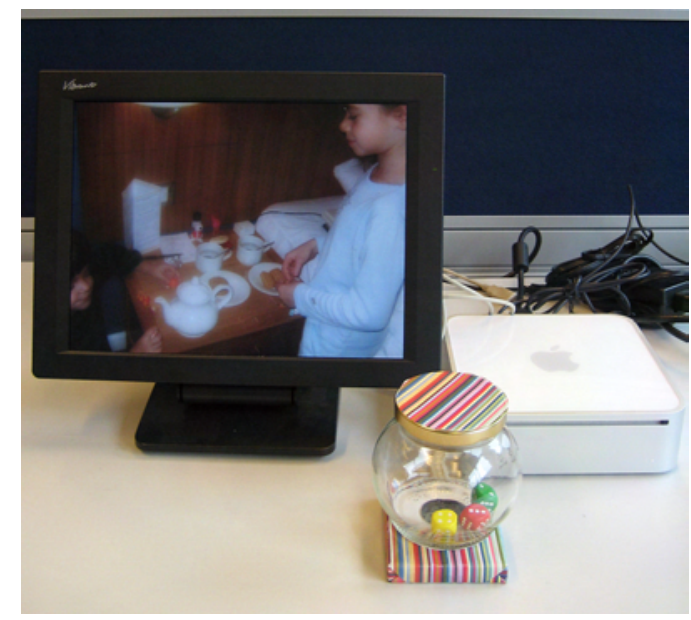

Figure 1: The PDS (Photo Display System)

\footnotetext{
1 Radio-frequency identification (RFID) is a wireless communication technology that allows computers, via an RFID reader to retrieve data stored on devices called RFID tags [Nath et al., 2006]
} 


\subsection{The photo presentation features}

Digital content. Following findings that serendipity is found to arise from random encounters with personally meaningful content, i.e. content imbued with the person's memories or personal associations [Leong, T. et al., 2008a; Nunes et al., 2009], the PDS will only contain people's personal collection of photos.

Randomness. Leong et al [2008a] assert that encounters with serendipity are more likely to arise when the random selection is unconstrained, i.e. when the shuffle algorithm picks tracks randomly and freely from people's entire music library instead of being constrained to only subsets of the library. Thus, the PDS application uses an algorithm that randomly draws photos from the entire photo library and not subsets of the library. Also, the PDS does not allow people to create subsets of their photo library.

Defamiliarization destabilizes the familiar by rendering it slightly strange, thus heightening our attention [Shklovsky, 1917]. In shuffle listening, the random presentation was found to act as a defamiliarization agent by tearing tracks from their known place and order, allowing listeners to encounter them under different contexts. This forces listeners into an interpretive stance, and in the process (at least during shuffle listening), may be nudged towards serendipitous discoveries [Leong, T. et al., 2008a]. However, random presentation alone may not be sufficient to defamiliarize people's photos because such photos may be more resistant to defamiliarization.

Unlike people's digital music library, photo libraries contain content that is (primarily) authored by the owner or members of the family. People's music library may also contain tracks that are not quite familiar, acquired on the go or shared from others, but people's photo libraries are more likely to contain known and familiar content. So we encouraged defamiliarization by using image treatment: distorting photos to make them slightly unfamiliar, thus problematizing perception but without altering them beyond recognition. We applied three different treatments using Photoshop to every individual photo. As a result, there were 4 versions of each photo in people's library (see figure 2). These were (i) the original (which was almost always in color), and its treated counterparts in (ii) black and white, (iii) sepia, and (iv) blurred. People's photo collection was treated prior to being loaded onto the PDS.

Temporality. Another challenge was that music is a temporal medium while photos are spatial. Levinson [1997, p. 20] notes when comparing encounters of music and painting (also a spatial medium); that whilst a synoptic perception (or beholding of the whole) is possible in painting, it is not possible to do so with the temporal medium of music. In other words, while people can perceive the entirety of the photo instantly, music reveals itself to the listener gradually over time. Thus people can easily and quickly recognize and apprehend the photos' content, i.e., what it is, who is in it, where it was taken, why was it taken (even when encountered unexpectedly). Of course people may be able to discern some much-loved tracks quite easily. However the number of such tracks within the growing size of people's digital music libraries is relatively low.

So, we designed the PDS application to reveal individual photos slowly over time. In addition, we also provided some 'temporal transition'. Instead of presenting a still image of each photo, the PDS starts from a random point of the photo and gradually reveals the image using a panning and zooming effect inspired by the Ken Burns Effect ${ }^{2}$. This gives the illusion of the photo sailing across the screen while other parts of the image are revealed over time. Once the photo is maximized and fills the screen, it is displayed for 6 seconds before a subtle crossover fade effect introduces the following randomly selected photo.

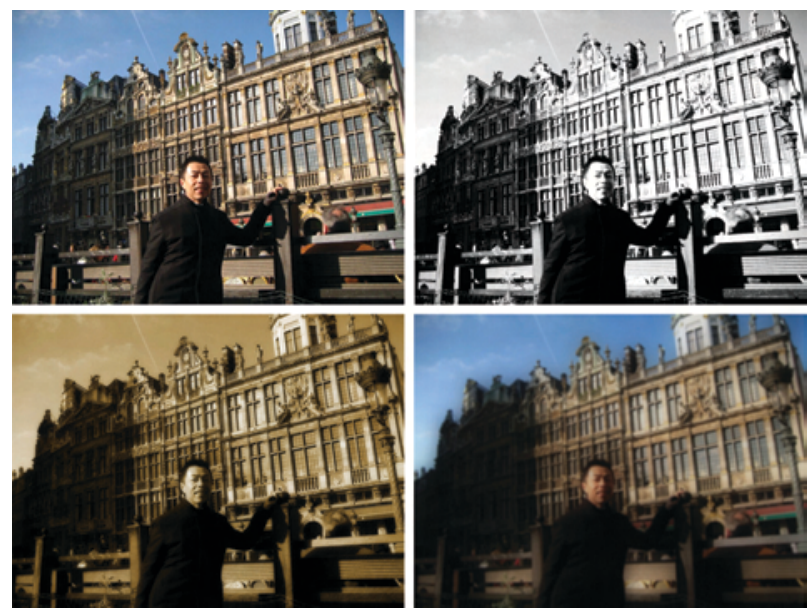

Figure 2: Photo treatments: original color (top left), black \& white (top right), sepia (bottom left) and blurred (bottom right)

\subsection{The dice-led input device}

While the PDS presents randomly selected photos continuously without any input from people, we added a dice-led input device to the system using Phidgets [Greenberg \& Fitchett, 2001]. This device does not confer any control beyond acting as a playful randomizer. It allows people to create an event that influences the PDS' behavior. Besides heightening people's awareness of randomness, we used this device to better understand how

\footnotetext{
2 The 'Ken Burns Effect' was credited in Apple's iMovie to the effect used extensively by Ken Burns in his documentary filmmaking.
} 
people made sense of their photos when encountering them in this random-led manner. Finally, we hoped that it might conjure up serendipitous connections so we could study the experience more closely.

The dice-led input device has two major components: (i) a container with three dice plus an RFID tag attached underneath (fig. 3) and (ii) a Phidgets RFID tag reader enclosed in a box which is connected to the PDS via USB (fig. 4). We will describe this device in action later.
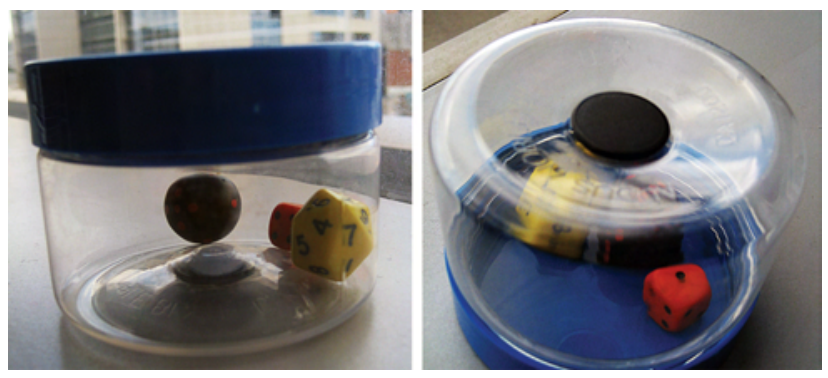

Figure 3: Container with dice (left) and revealing the RFID tag stuck to the bottom (right)

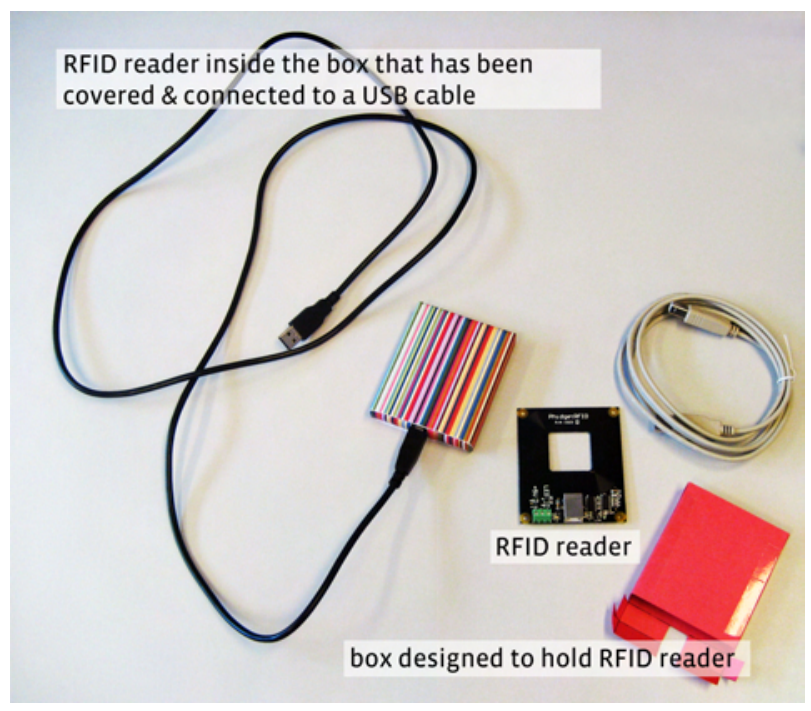

Figure 4: RFID tag reader with USB connection

\subsection{The PDS in action}

The PDS has two modes: passive (default mode) and interactive. The PDS launches automatically when the computer is switched on (in the passive mode). The RFID reader is constantly connected to the computer via USB. The container of dice (with the RFID tag) rests on top of the reader (fig. 1).

Passive mode. The PDS randomly chooses photos from people's library, presenting them, one at a time. This continues until people interrupt the system either by switching off the computer or by picking up the dice container.
Interactive mode. People can interact with the PDS by picking up the container to shake the dice. This triggers a series of (almost) simultaneous events. Once the tag reader senses that its connection with the tag is disrupted, its software stores the current time. Simultaneously, a signal is sent to the PDS ceasing the passive presentation of photos. Instead, two fast slide shows consisting of the visual shuffling of a series of randomly selected photos from the library at the rate of 2 photos per second is seen on the display. This continues until the container of dice is returned to its resting place (on top of the reader). Once the connection between the tag and reader is restored, the software again stores the current time and sends a signal to the PDS to decelerate the visual shuffling to a complete stop within 5 seconds. The difference between the two times, i.e. the duration of the dice roll (in milliseconds), is then used by the PDS software to seed a random calculation to select two photos. The algorithm uses the pseudorandom number generator provided in the .Net Framework, though we also explored using an I Ching algorithm in reference to Cage's work on chance operations [Cage, 1968] to select the two photos. These photos are presented in its entirety and left on the screen for 10 seconds, side by side, remaining still on the screen, after which the passive mode takes over once again.

\section{PDS DEPLOYMENT AND EMPIRICAL STUDY}

The PDS was deployed in three households. It was placed where members of the household were most likely to gather and spend time together. Households were asked to leave the PDS on all the time, if it was at all possible. Table 1 summarizes the details of the household members, their relationships to each other, the age of the children, the size of the photo library housed in the PDS and the deployment period. All names have been anonymized.

Table 1: Details of households and deployment

\begin{tabular}{|l|l|l|l|l|}
\hline Family & Members & $\begin{array}{l}\text { Library } \\
\text { size }\end{array}$ & Location & $\begin{array}{l}\text { Deployment } \\
\text { period }\end{array}$ \\
\hline 1 & $\begin{array}{l}\text { Michael \& } \\
\text { Jane, } \\
\text { Sons: Wesley } \\
\text { (9), John (6) }\end{array}$ & photos & Kitchen & 3.5 wks \\
\hline 2 & $\begin{array}{l}\text { Tom \& Mary } \\
\text { Daughter: } \\
\text { Megan (16), } \\
\text { Son: Ben (15) }\end{array}$ & $\begin{array}{l}20,000 \\
\text { photos }\end{array}$ & Lounge & 3 wks \\
& $\begin{array}{l}\text { Robert \& Minh } \\
\text { (engaged) }\end{array}$ & $\begin{array}{l}4,000 \\
\text { photos }\end{array}$ & Lounge & 2.5 wks \\
\hline 3 & & & \\
\hline
\end{tabular}

\subsection{Data collection}

We left a digital voice recorder next to the computer with each household to record their comments, 
reflections and experiences of the PDS. Only Household 1 used this consistently. In part this was because the young boys found listening back to their own voices a novelty. Their enthusiasm infected their mother. The other households were less keen. Household 2 preferred to sit and chat about the system during the period of the study rather than make recordings. They felt that the use of the recorder would hinder the experience. But given the reasonably short duration of the study and the fact that they shared and reflected upon their own experiences with each other throughout the study meant that they were able to remember much of what happened. These were recounted during the interview. For Household 3, interactions with the PDS were much more solitary. Robert was away a lot for work during the deployment. When I went to pickup the recorder, Minh told me that she used it sparingly because she hated the sound of her own voice but instead made notes on her computer.

\subsection{Interview}

The interviews lasted from between 1 hour to 2 hours and were recorded digitally. This semistructured group interview focused primarily upon getting at participants' experiences with the system. For instance, we sought to explore the range and types of people's experiences when encountering their photos in a random manner and also encounters with treated version of those photos. We also inquired if anyone encountered moments of serendipity, and if so, explored how such encounters came about, circumstances of those encounters, and what the experience was like for the person. All recordings were transcribed into Word documents. Transcripts of each household interview ranged from 3000 to 6,000 words.

\subsection{Data analysis}

We analyzed the data of each household individually, one at a time. Before that, we read through the transcript(s) many times in order to get a sense of the data. Given the aim of this study, the first pass through the data involved identifying passages that described encounters of serendipity with the PDS. Working outwards from these instances, we examined the data as to whether the particular features influenced people in encountering serendipity as well as people's viewing experiences in general.

\section{FINDINGS}

Participants' data is labeled as follow: $\mathrm{H} 1, \mathrm{H} 2$ and H3 represents each household respectively. Quotes from participant-directed audio recordings is labeled ' $R$ ', while interview quotes labeled 'l'.

\subsection{Serendipity}

Every household reported encountering serendipity during the study period. Broadly speaking, serendipity was encountered in three different ways: Type 1 , when a randomly presented photo was found to resonate with people's inner states: what they were thinking about, how they were feeling and so on, Type 2 when people found a meaningful conjunction between the random photo with something happening in people's surroundings and Type 3 when people found a meaningful connection between two random photos. Besides these connections with the photos, the serendipitous encounters also led people to certain unanticipated realizations or for some, a trigger for action.

\section{Type 1: Random photos and inner state}

"I am thinking a lot about my parents today. They will be visiting soon (from Canada). My dad was diagnosed with dementia last year and he recently had a fall. Photos of my parents kept coming up every minute. I noticed the first one and then more kept coming up. It was quite serendipitous." Jane, mother, $\mathrm{H} 1, \mathrm{R}$

Jane explained that,

\begin{abstract}
"It comforts me because it felt like the system was in sync with me. Sort of feel that it will be all right and that $\mathrm{I}$ am sending mental messages and the system responded especially after that moment I rang my parents. But oddly, something else was serendipitous because it also made us think of Michael's dad [Jane's father-in-law] who fell quite ill that week. Although it was not his pictures coming up, it reminded us of his father. So we rang them too." Jane, mother, $\mathrm{H} 1, \mathrm{I}$
\end{abstract}

Interestingly, it is the preponderance of photos around the same subject matter, i.e., Jane's parents (instead of just a single photo of them) that led to Jane's serendipitous moment. The multiplicity caught and reinforced Jane's attention thus fuelling Jane's sense making of the moment. She felt comforted and glad as a result of the serendipitous experience and was spurred to think of her in-laws. The moment even urged her to phone them.

Megan also reported this type of serendipity when she noticed the frequency of similarly 'themed' pictures that spoke to what was in her mind.

"Ben told me he had a dream about one of our cousins. So I came down[stairs] and was thinking about our cousin and a whole lot of them [photos of the cousin] came up. So I was like HEY! It is good when you are thinking about something and then you notice the ones related to it comes up. It is telepathic in a weird sort of way. It almost seems that the system knows, 
yeah. I was feeling quite special, especially the first one. It makes feel excited and I wish someone were sitting next to me then so that I could point it out. I was quite chuffed because there were like 10 photos in like 5 minutes. I was like whoa!" Megan, daughter, H2, I

\section{Type 2: Random photos and an event in the viewer's world}

Serendipity was encountered via the PDS (albeit not frequently reported during the study period) when people found meaningful conjunctions between the randomly presented photo and something happening in the person's environment/world.

\begin{abstract}
"I was thinking of my cousin and was reminding myself to call them when I noticed that there were more photos of Germany because he is living in Germany. There were more photos of his kids. And then soon after that, he called. That was serendipitous. Made me ponder our relationship." Minh, H3, I
\end{abstract}

When Minh was probed further as to how she felt when she realized that it was his cousin calling,

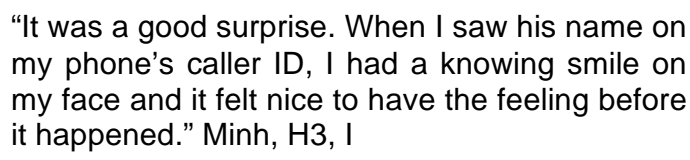

Type 3: Multiple photos. Serendipity was observed during the use of the dice input device. Whilst playful, the shaking of the dice container appeared to encourage and heighten people's anticipation and curiosity that primed their sense making of the photo shuffle.

\begin{abstract}
"I just shook the dice. How odd, when it shuffled, it came up with Admiral Pierce's wife on one side and opposite, a painting of Winston Churchill. This is quite interesting because they are obviously linked. Admiral Pierce would have taken orders from the Canadian army, working with the British Navy, ultimately organized by Winston Churchill. Wonderful! Never thought of it like that." Jane, mother, H1, R
\end{abstract}

Only Jane could have declared these two pictures as "obviously linked" thus making a serendipitous connection. This is because of her family's connection with the Admiral, and that Jane's mother was researching a book about the Churchill family. In fact, the photo of Churchill was in Jane's library because she had scanned it for her mother's research.

Next, we examine the various PDS features to see how they engaged viewers whilst priming them with resources that can be used to make serendipitous connections. A key role is the random presentation mechanism.
The random presentation engaged viewers by heightening attention and drawing people more deeply into their photos. The unpredictability added a sense of excitement, a bit of edge, and in most cases, made the experience more enjoyable.

\begin{abstract}
"The unexpectedness of how that happens is wonderful. Makes the pics more fun to look at. It puts the experience out of your control, which is interesting because you are in a journey you have not chosen. I quite like that. It's very nice to sit around, something pops up you didn't choose, so it prompts more of those, "oh did you remember that we did that?" Ben, son, $\mathrm{H} 2$, I
\end{abstract}

The random presentation constantly mixed things up. This meant that viewers were exposed to great variety of evocations and emotions. In fact, a single photo from a particular collection or occasion could serve as a 'memory placeholder'- a form of spatial and temporal compression [Middleton \& Brown, 2005 , p. 190] - that evokes the associated event or memories. For Mary, this experience had a meditative effect especially when she sat and viewed the photo display on her own (with a cup of tea) during occasions when she had the house to herself during the day.

\begin{abstract}
"You can just think fluidly...these photos and these memories just rush back and you take a couple of minutes just thinking about it. It's like a daydream when you are alone watching it moves from one thing to another. You are with your own thoughts and you are carried along in an unexpected journey. Quite relaxing." Mary, mother, $\mathrm{H} 2$, I

"It can take you from one emotion to another, from something really funny to something that leaves you reflecting about things." Minh, H3, I
\end{abstract}

The random delivery ripped individual photos from their 'group' or collection. This disrupted the sense of chronology and contextual associations people have imbued onto the photos. As a result, evocations encountered not only potentially crashed across time, they also move fluidly forwards and backwards in time. Besides surprise, this led to a refreshed view of the photo. Megan, borrowed the term 'genre' from music, to describe grouped photos in digital photo libraries,

\footnotetext{
"Genre is similar to say, Grand Canyon. When it skips across genres, I find that I look at the photos more because while photos of the Grand Canyon is lovely, after a while you just stop looking at them because they look the same don't they? But when it skips from say a photo of Grand Canyon to something recent, I couldn't anticipate it. It is one memory that 10 photos of Grand Canyon can prompt whereas for this is more intriguing...It carries you further and to many more places." Megan, daughter, H2, I
}

\subsection{The influence of randomness}


The random presentation intrigued viewers. Having to keep guessing was enjoyable. This sustained viewers' engagement and encouraged reflection as different evocations from each randomly presented photo took them to different places, and emotions.

\begin{abstract}
"It's so different from choosing pictures, like, well now we are going to look at this or that. If you knew what kind of genre the pics are, then you would have some guesses to what pictures will come up but would not be surprised. This is spontaneous and more fun." Megan, daughter, $\mathrm{H} 2$, I

"I really didn't expect to see this picture. I am reminded of things that my family has done and things that I have done. It's easy to forget what it felt like when you were there. It puts you back in touch with your past." Ben, son, H2, I
\end{abstract}

\subsubsection{Making sense of randomness}

The dice-led device allowed us to further glimpse how people made sense of the randomness.

\begin{abstract}
"It makes me ask questions like, why do I think of that? Of the thousands of photos, why these two? How does my mind connect these two? And if they are connected, you are fine, if they are not, you are stumped thinking why the dice brings to mind these two photos." Megan, daughter, $\mathrm{H} 2$, I
\end{abstract}

Most searched hard to discern any possible (and plausible) links or meaning that might connect them together. People reflected upon what they knew about the photos: delving in one's memories about the subject of the photos, traversing time and space, in an effort to (and often creatively), construct a connection between the two photos. People were found to be extremely creative and eager to make even the most tenuous links in order to find a (personally plausible) connection between the two randomly presented photographs.

\footnotetext{
"It made me contrast, compare, to think why and try and find links between them." Tom, father, $\mathrm{H} 2, \mathrm{I}$

"One of them came up with pine forest and one of my mum. That led to thinking about our old house in America... how does that relate? Wait...oh yes, the connection is that mum likes forests and so the two photos fit!" Megan, daughter, $\mathrm{H} 2$, I
}

In addition to supporting deeper immersion and reflexivity, the random presentation could also defamiliarize people's perceptions of their photos.

\subsection{Defamiliarization}

Encountering familiar photos ripped from their known grouping or genre, their chronology and context can surprise as well as potentially defamiliarizes viewers' perception of their photos.
What they thought they knew very well has become tainted with ambiguity. As a result, they are forced into an interpretive stance, where they tried to discern what they knew about the photo.

"If you were expecting something, you do look for it, you come away with the idea because you go with the set idea but this way it introduces many different themes, and pictures ad memories and sometimes we can't remember where it was taken and we try to find some geographic landmark." Michael, father, H1, I

"Because I didn't choose the photo, it alters your preconception of it. You just saw a photo from one time and place and suddenly a completely different one. So it forces you to look at it, to work out what it is." Jane, mother, $\mathrm{H} 1, \mathrm{I}$

But what appeared to heighten this defamiliarization were the Ken Burns effect and the photo treatment.

5.3.1 Ken Burns presentation \& Photo treatment Not revealing the entire photo instantaneously built anticipation, making it fun and mysterious.

\begin{abstract}
"Apart from random, the way it scans through the pic as it slowly reveals something, builds up anticipation and then it's not what you expect. It's like peeping through a keyhole of your own thoughts and you look through. We are now waiting to see what might happen next." Tom, father, $\mathrm{H} 2$, I
\end{abstract}

Altering a color photo into sepia, black and white or blurred, rendered something personally familiar and taken for granted, slightly unfamiliar. This lured people's interest, increased their involvement and sense making.
"The alterations of the photos are nice because it makes it different, changed in a way and it just makes you look at the photo a little differently." Minh, H3, I
"You are racking your mind about what it is and you can't because it's oddly familiar yet changed." Tom, H2, I

This thwarted people's immediate urge to finalize the meaning of the photo, i.e. to apprehend and know the photo thus rendering it closed off from further interpretation. Defamiliarized, people's perception of what they knew could be refreshed.
"If the entire photo just popped up showing everything, you'd quickly make up your mind and maybe even stop looking. This way it engages you more because some of them ...it's fun, and you are left guessing, and suddenly you are there in the moment. It's now a richer memory. " Robert, H3, I

Interestingly, what the viewer had 'learnt' from past experiences about the conventions and 
connotations of different treatments of photographs played into the defamiliarization.

\begin{abstract}
"I'm a black and white person because it's more artistic. When the black and white photos come up, I am highly aware of it. It makes me look at it more. I knew that it was in color and it forced me to re-examine it." Jane, mother, $\mathrm{H} 1, \mathrm{R}$

"You actually notice the individual more when there are people in it, there's a real focus that you really look at the person more when it's in black and white." Michael, father, $\mathrm{H} 1, \mathrm{I}$
\end{abstract}

Blurring also piqued viewers' attention, although with some it could be irritating as viewers tried hard to work out what it was.

"I was trying to adjust the monitor because it's really blurred. I don't think the blurring was a good idea. It was really irritating but it got our attention because we were all sitting around looking at it each trying to work out what it was." Michael, father, $\mathrm{H} 1, \mathrm{I}$

But Minh liked the blurring because it transformed a mundane photo into something else.

"Well, the blurry ones kind of look like glamour photos taken in professional studios, you know the ones with Vaseline lens. It was nice to see it transformed so differently. Gives it a different meaning." Minh, $\mathrm{H} 3, \mathrm{I}$

Viewers' understanding, preconception, and prior exposure to particular photographic conventions also influenced their sense making. Here, mood was also used to refer to this visceral connection.

"...somehow it [the treated photograph] takes you into a different mood. It brings a different kind of mood." Robert, H3, I

By including treated photographs into the PDS, we also added to the variety of styles, invigorating people's engagement with photographs they encountered:

"So that's added variety, spicing it up, not just the photos we put in." Jane, mother, $\mathrm{H} 1$, I

In the next section, we discuss just how by (i) fostering a state of engagement, immersion, anticipation and surprise and (ii) supplying resources like varied evocations in new order and defamiliarized photos, the designed features of the PDS not only supported but also under certain circumstances nudged viewers towards encountering serendipity.

\section{DISCUSSION}

The use of people's own photo collection meant that they are regaled with familiar and personally meaningful memories and emotions. But the random presentation ripped photos from their chronology and contexts creating new ordering and juxtapositions of evocations, memories and emotions. In effect, this provided viewers with resources for sense making: a new ordering of photos whereby potentially new narratives could be constructed or perhaps even serendipitous connections to be discerned. This also provided people the opportunities to notice meaningful patterns such as a preponderance of particular photos or new narratives emerging from the juxtaposition of disparate photos. At the same time, the variety of evocations arising from the photos traversing fluidly across time also piqued and heightened viewers' attention. This afforded viewers with more opportunities to discern meaningful and again, even serendipitous connections, either from amongst the disparate photos or connections to their current state and setting. Similarly, adding temporality using the Ken Burn's presentation style led to intrigue, built suspense and was effective in actively attracting viewers' attention, heightening their engagement and led to deeper immersion. The effect was fun and led to richer acts of remembering.

On the other hand, the slow reveal aspect of the Ken Burns effect was able to taint the photos with ambiguity and contribute to the their defamiliarization. Compounding this were the treated photos. What was personally familiar and known was rendered slightly strange, and potentially unfinalized, opened to re-interpretation. For Bakhtin, this unfinalized state is filled with surprisingness, potentiality, freedom, and creativity [McCarthy \& Wright, 2004, p. 69]; where people, through their sense making could forge new insights or connections.

Thus defamiliarization urged people to make sense of photos that they knew but which have now momentarily eluded them - a relational and dialogical process whereby people's past, present and imagined experiences are brought together to look at a situation in order to make sense of it [McCarthy \& Wright, 2004]. This saw people attempting to discern connections with the photo(s) that had been rendered opened to reinterpretation (albeit temporarily), such as with what they were thinking or feeling, what's around them or what they have previously seen on the monitor. And since these were ultimately their own photos, it is likely that personally meaningful connections could be forged or realizations made, some of which are potentially serendipitous. In fact, as we will explain next, people were often found to willingly make connections that appear tenuous, creatively joining the dots to forge something meaningful. 
What people brought to the moment can also nudge the sense making process towards serendipity. The daily ins and outs of viewers' family lives, conversations, activities and so on meant that people's minds were often simmering with various thoughts that could be drawn upon during their act of sense making as resources that can potentially connect with a randomly presented photo or photos. For example, Jane's mind was already primed with thoughts of her parents and their impending visit. Thus it is understandable why she could connect photos of her parents meaningfully and serendipitously with her inner states. And since photos of her parents would naturally be found in her photo collection, it is likely that photos of them would be presented. So whilst the PDS randomly supplied already meaningful content to Jane, she was at the same time ready to seek out connections. This shows just how by fostering certain conditions and providing meaningful resources, the PDS is in part nudging people towards serendipity. All is left, is for people to be engaged and attentive: whereby through acts of creative sense making discover serendipity.

\section{CONCLUSION}

This investigation contributes towards understanding how serendipity could arise and how it could be supported during people's interactions with digital photos. Through a design-led approach: by designing a PDS with particular features of random delivery, temporality and defamiliarization, we explicated how serendipity can arise during photo viewing and captured a glimpse of its potentially rich character. In fact, we found that these features could also be used fruitfully when considering how future technologies could be designed in general to support people in more engaging and immersive experiences when interacting with personal digital photos. More interestingly, our findings concur with Leong et al's [2005; 2006; 2008a; 2008b] exploration of shuffle listening-led serendipity (albeit with some designerly inspirations). Here, we demonstrate (for the first time) how these features could be harnessed to actively influence and even nudge people to encounter serendipity. With people's hopes, desires, worries, and fears simmering in their consciousness, meaning seeking people go about their quotidian lives primed and ready for resolution, answers or even confirmations. So by harnessing technology to evoke assortments of familiar and meaningful memories unexpectedly, and rendered slightly strange; while juxtaposed with other dislodged memories, we can potentially provide a congenial scaffold whereby people (if they so choose and are engaged), can actively and creatively forge their own meanings thus nudged to discover personally meaningful connections and experience serendipity.

While we don't claim that this is the only approach towards supporting for serendipity with digital photos, it is nevertheless a productive basis for further investigations of this technology-mediated serendipity. After all, this is a design prototype and there are various aspects and influences that can be further explored in the future. For example, investigating the influence of library size, and the influence of adding or removing photos upon people's viewing experiences. Another is the length of the deployment. The amount of time that families spent with the PDS in this study was relatively short. It will be interesting to see how their viewing experiences might evolve over time and in turn how this might influence their capacity to encounter serendipity can be explored. Given that most of our participants' photo collections were taken in the digital format, what they encountered were more recent memories. Thus, it will be fruitful to explore viewers' experiences if their photo collection includes photos taken prior to digital photography, i.e. a collection that spans a broader timeline. Tackling this (and investigating other influences) can certainly contribute to an even more nuanced understanding of this serendipity.

Finally this work can provide a basis for other efforts to investigate how we might support for serendipity during people's interactions with other digital media. Future work could explore how people's sense making of the elements of randomness, defamiliarization, and temporality may nudge people towards serendipity when interacting with other digital content such as videos and texts.

This work also contributes to demonstrating how defamiliarization can be harnessed to engage people during their interactions with digital content. Unlike some who approach defamiliarization as something designers can use for self-reflection in order to inspire design [Bell et al., 2005], here, we demonstrate how defamiliarization can be supported by technology - through the use of random presentation, temporality and photo-filters.

Van Andel [1994] concludes that serendipity is not something that can be planned for or programmed by computer. Extending his conclusion, we can also concur that any attempt to design serendipity is to extinguish the phenomenon itself. However, whilst our investigation suggests that we cannot design serendipity, there is definitely potential for designers to provide a congenial scaffold whereby this engaging and enigmatic experience may be discovered opportunistically during people's interactions with digital content. 


\section{ACKNOWLEDGEMENT}

This research was conducted while the first author was interning at Microsoft Research Cambridge, UK.

\section{REFERENCES}

Austin, J. H. (2003). Chase, chance, and creativity: The lucky art of novelty. MIT Press Cambridge, USA.

Bell, G., Blythe, M., \& Sengers, P. (2005). Making by making strange: Defamiliarization and the design of domestic technologies. ACM Transactions in Computer-Human Interactions, 12(2), 149-173.

Bentley, F., Metcalf, C., \& Harboe, G. (2006). Personal vs commercial content: the similarities between consumer use of photos and music. In Proc of $\mathrm{CHI}$, Montreal, Canada, 667-676.

Bull, M. (2006). Investigating the culture of mobile listening: From Walkman to iPod. In K. O'Hara \& B. Brown (Eds.), Consuming music together: Social and collaborative aspects of music consumption technologies (pp. 131-149): Springer. Dordrecht, Netherlands.

Cage, J. (1968). Diary: How to imporve the world (you will only make matters worse). In M: Writings '62-'72 (first published 1973): Wesleyan University Press.

Cosley, D., Akey, K., Alison, B., Baxter, J., Broomfield, M., Lee, S., et al. (2009). Using technologies to support reminiscence. In Proc British HCl, Cambridge, UK, 480-484.

Czerwinski, M., Gage, D. W., Gemmell, J., Marshall, C. C., Pérez-Quiñones, M. A., Skeels, M. M., et al. (2006). Digital memories in an era of ubiquitous computing and abundant storage. Communications of the ACM, 49(1), 44-50.

Durrant, A., Alex, S. T., Frohlich, D., Sellen, A., \& Uzzell, D. (2009). Photo displays and intergenerational relationships in the family home. In Proc British HCl, Cambridge, UK, 10-19.

Greenberg, S., \& Fitchett, C. (2001). Phidgets: Easy development of physical interfaces through physical widgets. In UIST'01, Orlando, Florida, 209218.

Harper, R., Rodden, T., Rogers, Y., \& Sellen, A. (Eds.). (2008). Being human: Human-computer interaction in the year 2020. Cambridge, UK: Microsoft Research Ltd.

Kirk, D., Sellen, A., Rother, C., \& Wood, K. (2006). Understanding photowork. In Proc of $\mathrm{CHI}$, Montreal, Canada, 761-770.

Leong, T., Vetere, F., \& Howard, S. (2005). The serendipity shuffle. In Proc of $\mathrm{OzCHI}$, Canberra, Australia, 1-4.

Leong, T., Vetere, F., \& Howard, S. (2008a). Abdicating choice: The rewards of letting go. Digital Creativity, 19(4), 233-243.
Leong, T. W., Howard, S., \& Vetere, F. (2008b). Choice: abdicating or exercising. In Proc of $\mathrm{CHI}$, Florence, Italy, 715-724.

Leong, T. W., Vetere, F., \& Howard, S. (2006). Randomness as a resource for design. In Proc of DIS, University Park, USA, 132-139.

Levinson, J. (1997). Music in the moment. Cornell University Press Ithaca, N.Y.

McCarthy, J., \& Wright, P. (2004). Technology as experience. MIT Press Cambridge, USA.

Merton, R., \& Barber, E. (2004). The travels and adventures of serendipity. Princeton University Press Princeton, NJ.

Middleton, D., \& Brown, S. D. (2005). The social psychology of experience. SAGE Thousand Oaks, California, USA.

Nath, B., Reynolds, F., \& Want, R. (2006). RFID Technology and Applications. IEEE Pervasive Computing, 5(1), 22-24.

Nunes, M., Greenberg, S., \& Neustaedter, C. (2009). Using physical memorabilia as opportunities to move into collocated digital photosharing. International Journal of Human-Computer Studies, 67, 1087-1111.

Petersen, M. G., Iversen, O. S., Krogh, P. G., \& Ludvigsen, M. (2004). Aesthetic interaction: a pragmatist's aesthetics of interactive systems. In Proc of DIS, Cambridge, MA, USA 269-276.

Roberts, R. M. (1989). Serendipity: Accidental discoveries in science. John Wiley \& Sons New York.

Shen, J., Shepherd, J., Cui, B., \& Tan, K.-L. (2009). A novel framework for efficient automated singer identification in large music databases. ACM Transactions on Information Systems, 27(3), 59-66. Shklovsky, V. (1917). Art as technique. In R. C. Davis (Ed.), Contemporary literary criticism: Modernism through poststructuralism: Longman. New York.

Shneiderman, B., Bederson, B. B., \& Drucker, S. M. (2006). Find that photo! interface strategies to annotate, browse, and share. Communications of the ACM, 49(4), 69-71.

van Andel, P. (1994). Anatomy of the unsought finding. Serendipity: Origin, history, domains, traditions, appearances and programmability. British Journal for the Philosophy of Science, 45, 631-648. 